OPEN ACCESS

Edited by:

Fatima Baltazar

University of Minho, Portugal

Reviewed by:

Stephan Jagger Guyenet

Self-employed, Seattle, WA,

United States

Miguel Luiz Batista Júnior

University of Mogi das Cruzes, Brazil

*Correspondence:

Yasmin Alaby Martins Ferreira yasminalaby@hotmail.com

Lila Missae Oyama

Imoyama@gmail.com

tPresent Address:

Raquel Munhoz da Silveira Campos,

Post Graduate Program of Interdisciplinary Health Sciences and

Department of Biosciences,

Universidade Federal de São Paulo

(UNIFESP), Santos, Brazil

Specialty section:

This article was submitted to

Clinical Nutrition,

a section of the journal

Frontiers in Nutrition

Received: 04 January 2019

Accepted: 10 May 2019

Published: 03 June 2019

Citation:

Ferreira YAM, Kravchychyn ACP,

Vicente SdCF, Campos RMdS,

Tock L, Oyama LM, Boldarine VT, Masquio DCL, Thivel D, Shivappa N, Hébert JR and Dâmaso AR (2019) An Interdisciplinary Weight Loss Program

Improves Body Composition and Metabolic Profile in Adolescents With Obesity: Associations With the Dietary Inflammatory Index. Front. Nutr. 6:77. doi: 10.3389/fnut.2019.00077

\section{An Interdisciplinary Weight Loss Program Improves Body Composition and Metabolic Profile in Adolescents With Obesity: Associations With the Dietary Inflammatory Index}

\author{
Yasmin Alaby Martins Ferreira ${ }^{1 *}$, Ana Claudia Pelissari Kravchychyn ${ }^{1}$, \\ Sofia de Castro Ferreira Vicente ${ }^{1}$, Raquel Munhoz da Silveira Campos ${ }^{2+}$, Lian Tock ${ }^{3}$, \\ Lila Missae Oyama ${ }^{1,4 *}$, Valter Tadeu Boldarine ${ }^{4}$, Deborah Cristina Landi Masquio ${ }^{5}$, \\ David Thivel ${ }^{6,7}$, Nitin Shivappa ${ }^{8,9,10}$, James R. Hébert ${ }^{8,9,10}$ and Ana R. Dâmaso ${ }^{1}$ \\ ${ }^{1}$ Post Graduate Program of Nutrition, Escola Paulista de Medicina, Universidade Federal de São Paulo, São Paulo, Brazil, \\ 2 Therapeutic Resources Laboratory, Department of Physiotherapy, Universidade Federal de São Carlos (UFSCar), São \\ Carlos, Brazil, ${ }^{3}$ Post Graduate Program of Nutrition, Universidade Federal de São Paulo, São Paulo, Brazil, ${ }^{4}$ Department of \\ Physiology, Escola Paulista de Medicina, Universidade Federal de São Paulo, São Paulo, Brazil, ${ }^{5}$ Centro Universitário São \\ Camilo, São Paulo, Brazil, ${ }^{6}$ Clermont Auvergne University, EA 3533, Laboratory of the Metabolic Adaptations to Exercise \\ Under Physiological and Pathological Conditions (AME2P), Clermont-Ferrand, France, ${ }^{7}$ CRNH-Auvergne, Clermont-Ferrand, \\ France, ${ }^{8}$ Cancer Prevention and Control Program, University of South Carolina, Columbia, SC, United States, ${ }^{9}$ Department \\ of Epidemiology and Biostatistics, Arnold School of Public Health, University of South Carolina, Columbia, SC, United States, \\ ${ }^{10}$ Connecting Health Innovations LLC (CHI), Columbia, SC, United States
}

Background and Aims: The prevalence of overweight and obesity consitutes a global epidemic and it is growing around the world. Food and nutrition are essential requirements for promoting health and protecting against non-communicable chronic diseases, such as obesity and cardiovascular disease. Specific dietary components may modulate inflammation and oxidative stress in obese individuals. The Dietary Inflammatory Index $\left(\mathrm{D} \|{ }^{\circledR}\right)$ was developed to characterize the anti- and pro-inflammatory effects of individuals' diet. Few studies have investigated the role of diet-associated inflammation in adolescents with obesity. The present study aims to investigate the effects of an interdisciplinary weight loss therapy on DII scores and cardiometabolic risk in obese adolescents and possibles correlations.

Methods: A total of 45 volunteers (14-19 years old) were recruited and enrolled for long-term interdisciplinary therapy including clinical, nutritional, psychological counseling, and exercise training. Adolescents had access to videos about health education weekly. Body composition and inflammatory and serum profiles were evaluated at baseline and after intervention. The food intake was obtained by 24-h food recall. Data was used to calculate energy-adjusted DII (E-DII) scores. Negative scores indicate an anti-inflammatory diet and positive scores indicates a pro-inflammatory diet. The sample was divided according to whether individuals increased or decreased E-DII scores after therapy. 
Results: After therapy the body mass index (BMI), body weight, body fat, abdominal, waist, neck, and hip circumferences decreased significantly. The mean of high-density lipoprotein cholesterol (HDL-c) increased after the therapy. There was found an improvement of inflammatory and cardiometabolic parameters. In exploratory analyses, this occurred mainly when the EDII improved.

Conclusion: Long-term interdisciplinary therapy combined with a health education website improved inflammatory serum markers in obese adolescents. Reduction in DII scores was associated with reduction of cardiometabolic parameters, suggesting that an anti-inflammatory diet may be an effective strategy to prevent and treat obesity and related comorbidities.

Trial: http://www.ensaiosclinicos.gov.br/rg/RBR-6txv3v/, Register Number: RBR$6 t x v 3 v$

Keywords: inflammatory diet, cardiometabolic risk, interdisciplinary therapy, obesity, inflammation

\section{INTRODUCTION}

Obesity is a multifactorial disease that requires interdisciplinary therapy and preventive strategies. Obesity-related comorbidities, such as diabetes and cardiovascular disease, currently represent the main costs incurred by health care systems $(1,2)$. The prevalence of obesity in children and adolescents is increasing worldwide. In Brazil, 20.5\% of adolescents between 10 and 19 years old are overweight and $4.9 \%$ are obese $(3,4)$.

Obesity is not just a matter of aesthetics and body image. It also decreases life expectancy and results in cancer, cardiovascular diseases, hypertension, diabetes, steatosis, characterizing metabolic syndrome; all together raising mortality risks $(2,5-7)$.

Adipose tissue releases adipokines that have specific functions, among them; adiponectin may attenuate inflammatory response and results in beneficial effects on cardiovascular disorders. However, studies indicate that obese individuals present lower concentrations of adiponectin, and concomitantly they are in a state of hyperleptinemia, favoring inflammation. The change in homeostasis and release of cytokines, characterize obesity as a condition of persistent low-grade inflammation, which is closely associated with obesity-related comorbidities (8-11).

Leptin is one of the main factors involved in the energy balance regulation. Obese patients show leptin-resistance that, in turn, can favor inflammatory state (12). In fact, leptin resistance in obese adolescents could influence an expansion of adipose tissue by increasing the release of leptin to supply the resistance, thus leading to problems with weight loss, and contributing to inflammation (13). Therefore, the adiponectin/leptin ratio is an interesting and pertinent indicator relating to adipose tissue dysfunction (14).

While food intake and eating habits are key factors for the promotion of health and prevention of weight gain and cardiovascular disease, some specific dietary components may modulate the inflammation and oxidative stress in obese individuals. Indeed, several studies have observed associations between inflammatory diets and comorbidities, such as obesity, metabolic disorders, intestinal dysbiosis, Parkinson's disease, cancer, depression, and bone weakness (15-23).

The Dietary Inflammatory Index (DII $\left.{ }^{\circledR}\right)$ was created in 2009 to quantify the effect of diet on inflammatory potential, which was further improved and validated by Shivappa et al. (24). A literature review in 2010 encompassing 1,943 papers studied the association between each of the six inflammatory markers (Interleukin-1 $\beta$, Interleukin-4, Interleukin-6, Interleukin-10, Tumor Necrosis Factor- $\alpha$, and C-reactive protein) and 45 food parameters. Negative scores suggest that the meal or ingested food may have anti-inflammatory effects, while positive values indicate pro-inflammatory effects (24). Specifically, among South American population, DII has been tested with colorectal and prostate cancers in Argentina and multiple sclerosis and metabolic syndrome in Brazil (25-27).

Previously, a pro-inflammatory diet has been validated with inflammatory markers among adolescents and has been shown to be associated with increased risk of childhood obesity, cardiometabolic risk, depression, and stress levels among adolescents (28-32). Accordingly, studies conducted in adolescents have indicated that elevated intake of sugarsweetened soft drinks, refined grains, processed foods, and low intake of vegetables and fiber may increase the incidence of premenopausal breast cancer and cardiometabolic risks $(30,33,34)$.

Interesting, multi and interdisciplinary programs have been conducted in adolescents to promote obesity management and long-term weight loss therapies have been shown to produce favorable changes in metabolic parameters and inflammation. While the effects of exercise training are pretty well described, it remains so far unclear how diet can influence obesity related inflammatory markers, such as, adiponectin and leptin in adolescents (11,35-39).

This study was designed to investigate the effects of an interdisciplinary therapy combined online with support (i.e., web-based health education) on inflammatory markers and to verify possible correlations between energy-adjusted dietary inflammatory index (E-DII) with inflammatory markers in obese 


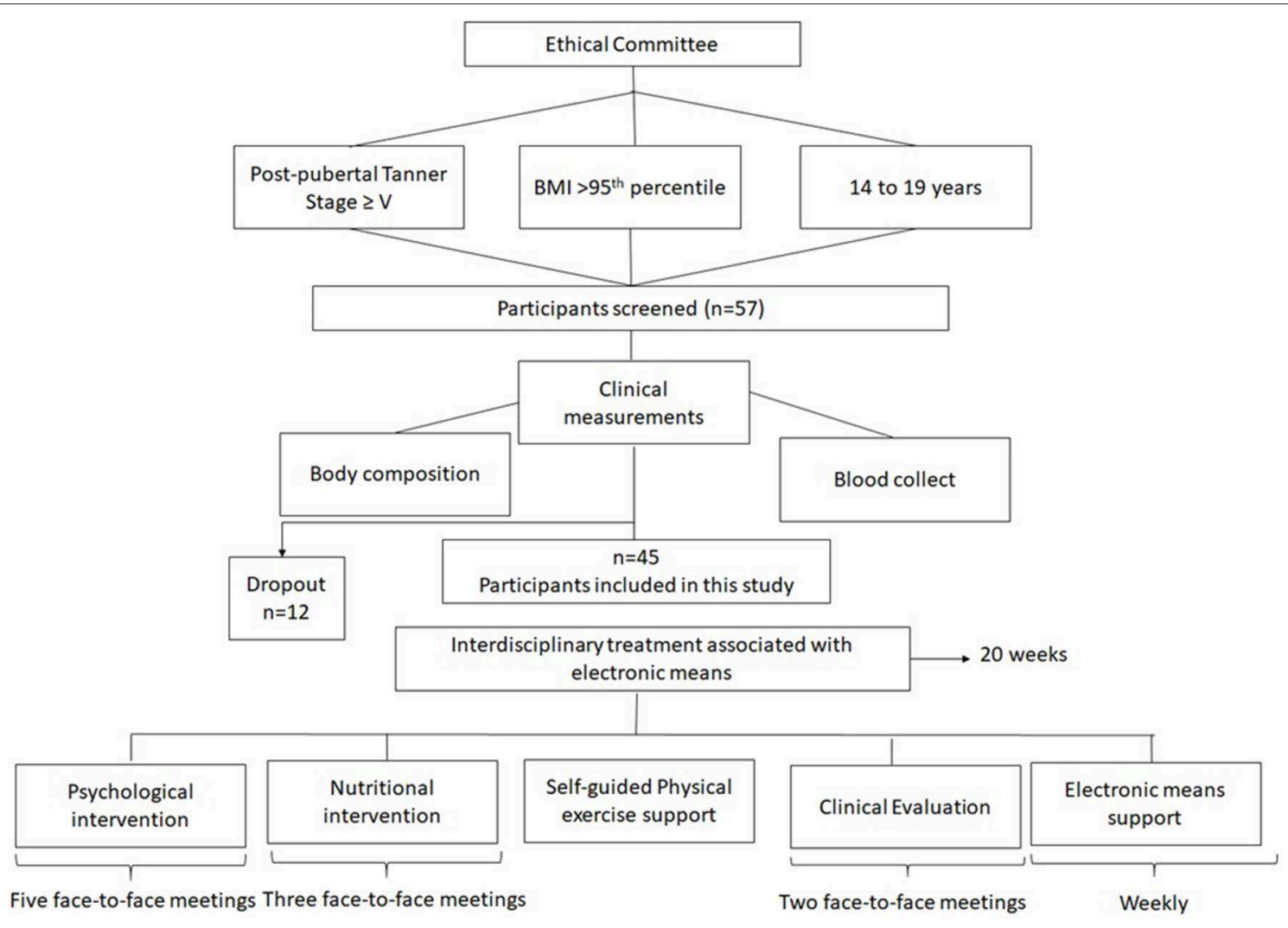

FIGURE 1 | Study design.

adolescents. We hypothesized that the therapy will improve body composition and inflammatory biomarkers, and that reduction of inflammatory biomarkers is correlated with changes in E-DII.

\section{MATERIALS AND METHODS}

\section{Population}

Forty-five pubertal obese adolescents (21 boys, 24 girls) were enrolled in this study. The study was announced in the media (journals, magazines, radio, and TV) and the adolescents were selected according to the inclusion and exclusion criteria. The inclusion criteria were a body mass index (BMI) $p>95$ th percentile (40); age between 14 and 19 years and post-pubertal Tanner Stage $\geq V$ (41). Exclusion criteria were cardiovascular disease, musculoskeletal problems that limited physical exercise, autoimmune diseases, genetic, metabolic or endocrine disease and chronic alcohol consumption and tabaco consumption and also who used drugs/medicaments and/or nutritional supplements that alter the metabolism during the last 6 months, and who did not have access to electronic means. Predicting a dropout of $\sim 20 \%, 57$ adolescents started the interdisciplinary therapy and 45 adolescents completed the intervention. The main reasons for the treatment dropout were job opportunities and school, followed by financial and family problems.

At initial interview the protocol was shared with the volunteers, who had received a parental consent form to participate in an interdisciplinary weight loss program. The study was developed in accordance with the Declaration of Helsinki and was approved by the Federal University to Sao Paulo ethics committee (\#0448/2018). Brazilian Clinical Trial registration number: RBR-6txv3v. The Figure $\mathbf{1}$ shows the study design.

\section{Anthropometric Measurements and Body Composition}

Body weight was measured using light clothes and barefoot on a Filizola ${ }^{\circledR}$ scale to $0.1 \mathrm{~kg}$ and capacity of $180 \mathrm{~kg}$. Height was measured using to a wall-mounted height board (Sanny ${ }^{\circledR}$ ) to the nearest $0.1 \mathrm{~cm}$. Body mass index was then calculated as body weight $(\mathrm{kg}) /$ height (meter) $)^{2}$. Waist circumference and abdominal circumference were measured with a flexible and inelastic tape. Body composition was measured by a Bioimpedance meter (BIA) and the basal metabolic rate was estimate based on body composition provide by the device BIODYNAMICS 310e (TBW $\left.{ }^{\circledR}\right)$, that has accuracy: Correlation of $R=0.98$ with Hydrostatic Weighing and accuracy of $\pm 1 \%$ in Resistance reading.

\section{Serum Analysis}

Blood samples were collected after an overnight 12 -h fast. Plasma insulin concentration was measured by radioimmunoassay. According to Schwimmer et al. (42), normal plasma levels of fasting insulin are lower than $17 \mathrm{uU} / \mathrm{ml}$. Plasma glucose 
was measured by an enzymatic method with a UV-Visible spectrophotometer and interpreted according to the established criteria by the American Diabetes Association (43). Total cholesterol, cholesterol fractions and triglycerides (TG) were analyzed using colorimetric comparative method. The cut-off limits for total cholesterol, LDL-c, HDL-c, and triglycerides were established according to the Brazilian Society of Cardiology (2013) (44). Insulin resistance (HOMA-IR) and insulin sensitivity (QUICKI) were estimated by evaluation model obtained using the formulas proposed by Matthews et al. (45) and Katz et al. (46), respectively:

HOMA-IR: Insulin fasted $(\mathrm{U} \mu / \mathrm{ml}) \mathrm{x}$ fasted blood glucose $(\mathrm{mmol} / \mathrm{ml}) / 22.5$

QUICKI: $1 /(\log$ fasting insulin $(\mathrm{U} \mu / \mathrm{ml})+\log$ fasting glycaemia $(\mathrm{mg} / \mathrm{dl})$

Adiponectin and Leptin were measured with enzyme-linked immunosorbent assay (ELISA) kit from R\&D Systems (Minneapolis, MN, USA).

\section{Interdisciplinary Therapy Associated With Web-Based Educational Health Approach}

Endocrinologists, nutritionists, psychologists, and exercise physiologists developed the interdisciplinary weight loss therapy. In the present investigation, the volunteers completed five face-to-face meetings, including individual and group sessions and during 20-weeks intervention they had access to videos with health topics based on e-book: Saber Emagrecer Dâmaso (47). Weekly, body weight was monitored by self-report and photo of their scales. The researchers advised that body weight should be measured on the same equipment to avoid variability among the equipment. Applications related to android mobiles were used to assist in the monitoring of physical exercise and nutritional prescription. Participants also were contacted by phone or email every week in order to motivate them and eventually answer their questions during the intervention period.

\section{Clinical Therapy}

Obese adolescents visited the endocrinologist at the beginning and after therapy to address the health and clinical parameters, including family history.

\section{Psychological Therapy}

Once a month, the psychologist discussed important themes about obesity, including, bulimia, anorexia, self-esteem, and anxiety in groups.

\section{Exercise-Training}

The physical activity level was monitored at the initial evaluation and every 5 weeks by the International Physical Activity Questionnaire (IPAQ) - short version (48). A self-guided method was applied, in which the adolescents chose exercises related to personal pleasure (49). In all face-to-face meetings a professional guided the adolescent to do exercise at least 3 times/week and duration of $1 \mathrm{~h} /$ session. Every week mobile apps were used to monitor the physical exercise.

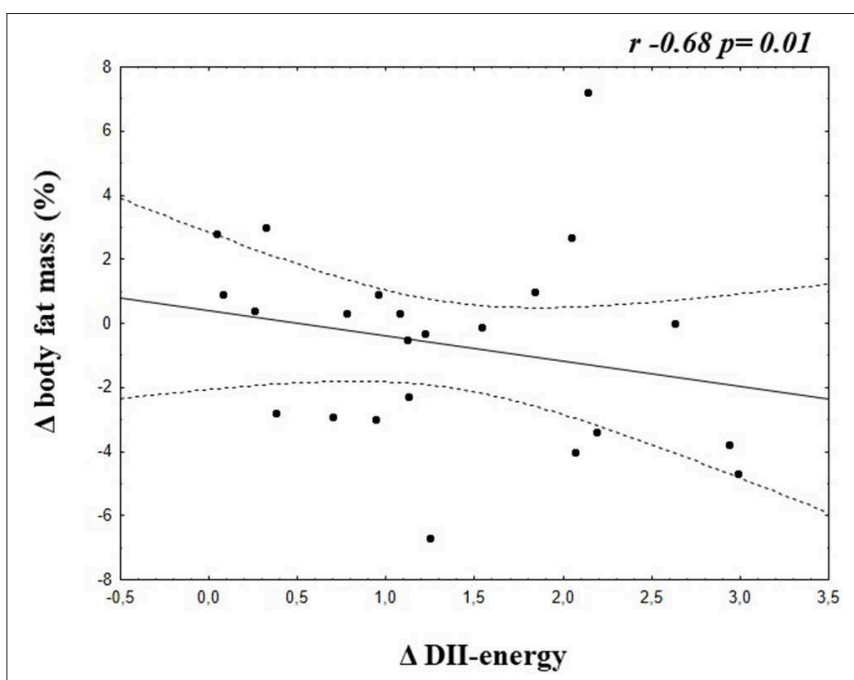

FIGURE 2 | Correlation between $\Delta \mathrm{E}$-DII and $\Delta$ Body fat mass of adolescents who increased the energy adjusted DII.

\section{Nutritional Therapy}

Different themes were discussed in personal sessions and online program weekly, including: food labels, diet and light foods, weight loss diets, good food choices on weekends, and celebrations. No supplements were recommended.

Daily energy intake was assessed using self-reported 24-h food recalls (24 HR) completed at baseline and at the end of the 20week intervention. Hypocaloric diet was prescribed according to age and gender, reducing caloric intake between 300 and 500 $\mathrm{kcal} /$ day, respecting the distribution of macronutrients proposed by the Institute of Medicine (50). The DIETSMART ${ }^{\circledR}$ program was used to analyze dietary intake and calculate nutrients intake to calculate the E-DII per 4,184 kJ (1,000 kcal) of food consumed (51). Lower values indicate an anti-inflammatory diet and higher values represent pro-inflammatory diet (24). Twenty -four nutrients were used to calculate the E-DII score (Carbohydrate, Protein, Fat, Fiber, Cholesterol, saturated fat, mono-unsaturated fat, poly-unsaturated fat, Omega3, Omega6, Niacin, Thiamin, Riboflavin Vitamins (A, B12, B6, C, D, E), Iron, Magnesium, Zinc, Selenium, Folic Acid, and Beta-carotene.

\section{Statistical Analysis}

Statistical analysis was performed using the program STATISTICA version 7.0 for Windows. The accepted significant value was $\alpha<5 \%$. Data normality was verified with the Kolmogorov-Smirnov test. Parametric data were expressed as mean \pm SD, and non-parametric data was normalized using $Z$-scores. To analyze the effects of intervention and difference between the groups according to the E-DII response: increased the E-DII or decreased E-DII, ANOVA for repeated measures (ANOVA two-way) followed by Fischer post hoc test were used.

Delta values $(\Delta)$ were used for the statistical analysis obtained from the difference between the after therapy and baseline values for each variable: $\Delta$ variable $=$ after therapy value-baseline value. Comparison of the delta values between the groups was 
performed by $t$-test independent by groups. Product moment; i.e., Pearson correlation coefficients were computed.

Using the $G^{*}$ Power ${ }^{\circledR} 3.0 .10$ program, it was obtained a sample size of 46 voluntaries, considering the statistical analysis realized by ANOVA for repeated measures (ANOVA two-way). The effect size 0.30 , power $80 \%$, based on E-DII and two groups and two times of evaluation (baseline and after 20 weeks of intervention).

\section{RESULTS}

\section{Effects of Therapy in Anthropometric Measurements and Body Composition}

The study population consisted of 45 adolescents. Assessment of anthropometric measurements and body composition at the beginning and after therapy are presented. The therapy was effective in reducing body weight, BMI, waist circumference, neck circumference, hip circumference and body fat mass $(\%$ and $\mathrm{kg}$; Table 1).

The subgroup of individuals that decreased E-DII showed significantly greater decrease in BMI, body fat mass ( $\mathrm{kg}$ and \%) and increase in fat free mass (\%) after therapy. The BMI, body weight $(\mathrm{kg})$, waist circumference, neck and hip circumference decreased in both groups (Table 1).

\section{Effects of Therapy on Biochemical Parameters}

Considering the biochemical parameters, only HDL-C was shown to significantly increase $(p<0.05)$ while all the other metabolic parameters remained unchanged. On the other hand, the subgroup with decreased E-DII had increase in QUICKI and reduction in glycaemia, VLDLc, triglycerides, and HOMA-IR. The therapy was effective in increasing the HDL-c even in the group that increased the E-DII.

\section{Effects of Therapy on Adipokines}

The leptin decreased significantly after the intervention, while adiponectin/leptin ratio increased in all adolescents. At baseline, the leptin concentration was statistically different between adolescents who increased and decreased the energyadjusted dietary inflammatory index (Table 2). Adolescents who decreased the E-DII reduced leptin and increase the adiponectin/leptin ratio after the therapy.

\section{Effects of Therapy on Diet Patterns}

The adolescents had significant decrease in energy intake after therapy, as well as reductions in dietary carbohydrate, lipids, and sodium intake. The glycemic load of the diet decreased (Table 3).

\section{Comparison the Effects of Therapy Between the Groups}

According to the deltas analysis it was observed that body fat mass and QUICKI were statistically different between adolescents who increased DII and decreased DII after therapy. No differences were observed in the deltas of adipokines (Table 4).

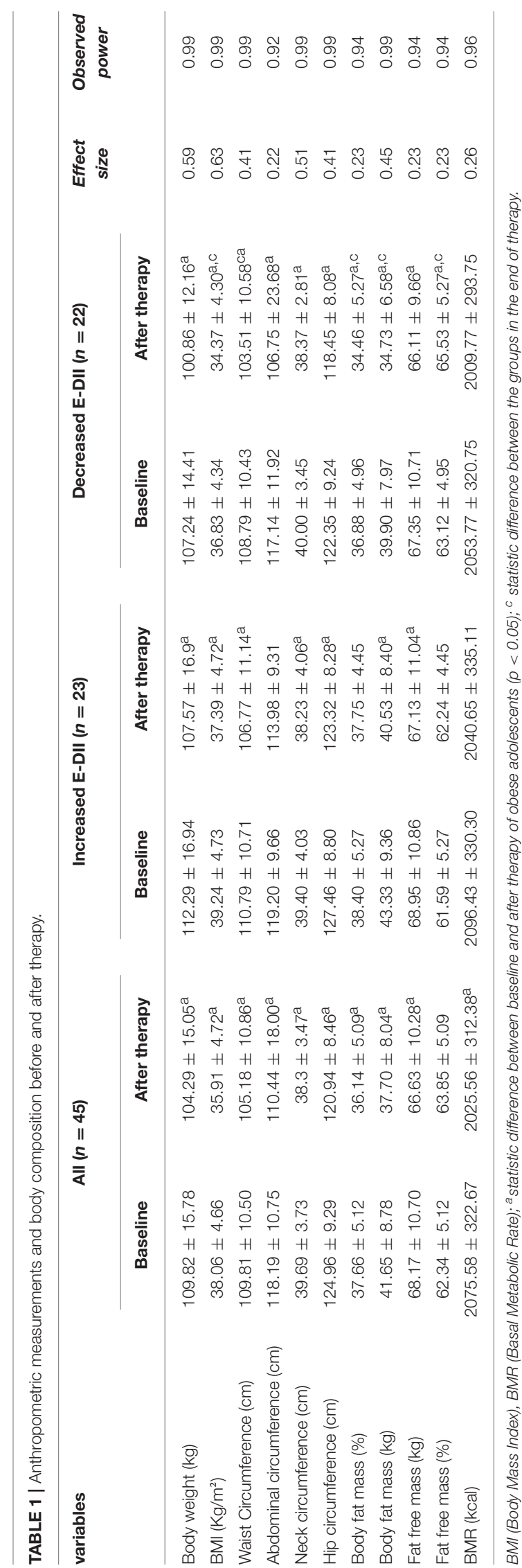


TABLE 2 | Biochemical parameters and adipokines of obese adolescents before and after therapy.

\begin{tabular}{|c|c|c|c|c|c|c|c|c|}
\hline \multirow[t]{2}{*}{ variables } & \multicolumn{2}{|c|}{ All $(n=45)$} & \multicolumn{2}{|c|}{ Increased E-DII $(n=23)$} & \multicolumn{2}{|c|}{ Decreased E-DII $(n=22)$} & \multirow{2}{*}{$\begin{array}{l}\text { Effect } \\
\text { size }\end{array}$} & \multirow{2}{*}{$\begin{array}{c}\text { Observed } \\
\text { power }\end{array}$} \\
\hline & Baseline & After therapy & Baseline & After therapy & Baseline & After therapy & & \\
\hline Glycemia (mg/dL) & $90.53 \pm 7.83$ & $88.73 \pm 7.51$ & $91.26 \pm 9.21$ & $91.04 \pm 6.71$ & $89.77 \pm 6.20$ & $86.32 \pm 7.67^{C}$ & 0.03 & 0.21 \\
\hline Total Cholesterol (mg/dL) & $164.53 \pm 34.58$ & $160.98 \pm 38.97$ & $160.78 \pm 39.93$ & $165.22 \pm 43.00$ & $168.45 \pm 28.34$ & $156.55 \pm 34.70$ & 0.009 & 0.09 \\
\hline $\mathrm{HDL}-\mathrm{C}(\mathrm{mg} / \mathrm{dL})$ & $40.51 \pm 8.43$ & $43.80 \pm 8.58^{a}$ & $41.52 \pm 9.37$ & $45.48 \pm 9.29^{a}$ & $39.45 \pm 7.39$ & $42.05 \pm 7.58$ & 0.23 & 0.94 \\
\hline Non-HDL (mg/dL) & $124.02 \pm 31.66$ & $113.80 \pm 36.41$ & $119.39 \pm 35.40$ & $113.13 \pm 38.84$ & $128.86 \pm 27.18$ & $114.50 \pm 34.59$ & 0.06 & 0.40 \\
\hline LDL-c (mg/dL) & $99.51 \pm 31.36$ & $93.80 \pm 32.91$ & $92.74 \pm 33.81$ & $92.35 \pm 37.76$ & $106.59 \pm 27.57$ & $95.32 \pm 27.77$ & 0.03 & 0.21 \\
\hline VLDL-c (mg/dL) & $24.44 \pm 9.18$ & $22.38 \pm 10.92$ & $26.52 \pm 10.50$ & $25.43 \pm 10.58$ & $22.27 \pm 7.17$ & $19.18 \pm 10.57^{c}$ & 0.03 & 0.25 \\
\hline $\mathrm{TG}(\mathrm{mg} / \mathrm{dL})$ & $121.96 \pm 45.76$ & $111.67 \pm 54.33$ & $132.57 \pm 52.38$ & $126.65 \pm 52.88$ & $110.86 \pm 35.50$ & $96.00 \pm 52.45^{\mathrm{C}}$ & 0.03 & 0.25 \\
\hline 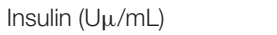 & $20.02 \pm 12.43$ & $17.83 \pm 13.03$ & $21.65 \pm 16.16$ & $21.45 \pm 16.44$ & $18.31 \pm 6.66$ & $14.04 \pm 6.59$ & 0.03 & 0.24 \\
\hline HOMA-IR & $4.51 \pm 2.84$ & $3.98 \pm 3.04$ & $4.90 \pm 3.66$ & $4.86 \pm 3.80$ & $4.10 \pm 1.58$ & $3.06 \pm 1.59^{\mathrm{C}}$ & 0.03 & 0.2 \\
\hline QUICKI & $0.31 \pm 0.02$ & $0.32 \pm 0.03$ & $0.31 \pm 0.03$ & $0.31 \pm 0.02$ & $0.31 \pm 0.02$ & $0.33 \pm 0.03^{a, c}$ & 0.08 & 0.51 \\
\hline Adiponectin $(\mu \mathrm{g} / \mathrm{mL})$ & $2.67 \pm 2.06$ & $3.64 \pm 3.27$ & $2.51 \pm 1.53$ & $3.90 \pm 3.74$ & $2.83 \pm 2.52$ & $3.36 \pm 2.75$ & 0.08 & 0.47 \\
\hline Leptin $(n g / m L)$ & $63.74 \pm 41.37$ & $49.80 \pm 41.38^{\mathrm{a}}$ & $75.78 \pm 47.96$ & $62.49 \pm 48.89$ & $51.17 \pm 29.20^{b}$ & $36.54 \pm 26.88^{\mathrm{C}}$ & 0.23 & 0.94 \\
\hline Adipo/lep & $0.06 \pm 0.05$ & $0.13 \pm 0.18^{\mathrm{a}}$ & $0.05 \pm 0.05$ & $0.11 \pm 0.12$ & $0.06 \pm 0.04$ & $0.16 \pm 0.22^{\mathrm{a}}$ & 0.18 & 0.86 \\
\hline
\end{tabular}

HDL-c, High-density lipoprotein cholesterol; LDL-c, Low-density lipoprotein cholesterol; VLDL-c, very low-density lipoprotein cholesterol; HOMA-IR, homeostasis model assessment insulin resistance; QUICKI, quantitative insulin-sensitivity check index. adipo/lep: adiponectin/leptin ratio;

Data are presented as mean (SD):

a statistic difference between baseline and after therapy of obese adolescents $(p<0.05)$;

${ }^{b}$ statistic difference between baseline of adolescents $(p<0.05)$;

$c^{c}$ statistic difference between the groups in the end of therapy. 
TABLE 3 | Energy intake, macronutrients, micronutrients, fiber dietary, and DII before and after therapy.

\begin{tabular}{|c|c|c|c|c|c|c|c|c|}
\hline \multirow[t]{2}{*}{ variables } & \multicolumn{2}{|c|}{ All $(n=45)$} & \multicolumn{2}{|c|}{ Increased E-DII $(n=23)$} & \multicolumn{2}{|c|}{ Decreased E-DII $(n=22)$} & \multirow{2}{*}{$\begin{array}{c}\text { Effect } \\
\text { size }\end{array}$} & \multirow{2}{*}{$\begin{array}{c}\text { Observed } \\
\text { power }\end{array}$} \\
\hline & Baseline & After therapy & Baseline & After therapy & Baseline & After therapy & & \\
\hline Energy Intake (kcal) & $1965.49 \pm 830.70$ & $1491.40 \pm 623.8^{\mathrm{a}}$ & $1894.09 \pm 901.66$ & $1532.70 \pm 639.21$ & $2040.14 \pm 763.37$ & $1448.23 \pm 619.22^{a}$ & 0.21 & 0.92 \\
\hline Carbohydrate (g) & $251.92 \pm 106.14$ & $181.06 \pm 67.14^{\mathrm{a}}$ & $245.10 \pm 120.69$ & $180.88 \pm 48.26^{\mathrm{a}}$ & $259.39 \pm 89.94$ & $181.25 \pm 83.69^{a}$ & 0.24 & 0.95 \\
\hline Protein (g) & $97.11 \pm 51.29$ & $81.24 \pm 57.8$ & $94.36 \pm 49.90$ & $90.36 \pm 76.14$ & $99.99 \pm 53.72$ & $71.70 \pm 27.22$ & 0.04 & 0.31 \\
\hline Fat (g) & $25.95 \pm 45.70$ & $14.22 \pm 10.17^{a}$ & $60.37 \pm 32.78$ & $50.46 \pm 44.21$ & $70.14 \pm 31.29$ & $49.28 \pm 33.93^{a}$ & 0.14 & 0.76 \\
\hline Saturated Fat (g) & $30.12 \pm 50.24$ & $12.92 \pm 8.16$ & $16.87 \pm 11.87$ & $15.55 \pm 10.01$ & $35.46 \pm 63.59$ & $12.83 \pm 10.40^{\mathrm{a}}$ & 0.06 & 0.39 \\
\hline Polyunsaturated fat (g) & $8.64 \pm 6.08$ & $6.09 \pm 4.87^{a}$ & $8.70 \pm 5.86$ & $6.31 \pm 5.87$ & $8.58 \pm 6.43$ & $5.86 \pm 3.69$ & 0.09 & 0.53 \\
\hline Fiber (g) & $19.68 \pm 13.4$ & $16.05 \pm 7.99$ & $23.03 \pm 13.90$ & $15.16 \pm 8.00^{\mathrm{a}}$ & $16.18 \pm 12.18$ & $16.99 \pm 8.07$ & 0.04 & 0.30 \\
\hline Dietary Cholesterol (mg) & $287.18 \pm 184.41$ & $236.5 \pm 185.32$ & $270.87 \pm 181.17$ & $263.88 \pm 209.76$ & $304.23 \pm 190.45$ & $207.87 \pm 155.51$ & 0.05 & 0.32 \\
\hline Sodium (mg) & $4172.73 \pm 3491.2$ & $2634.38 \pm 2264.83^{a}$ & $4389.47 \pm 3824.32$ & $3205.96 \pm 2690.59$ & $4150.68 \pm 3191.44$ & $2036.83 \pm 1559.32^{a}$ & 0.22 & 0.93 \\
\hline E-DII & $1.16 \pm 1.52$ & $0.83 \pm 1.49$ & $0.24 \pm 1.19$ & $1.57 \pm 1.31^{\mathrm{a}}$ & $2.21 \pm 1.20$ & $0.06 \pm 1.27^{a, c}$ & 0.11 & 0.66 \\
\hline Glycemic Load & $82.58 \pm 51.36$ & $59.82 \pm 35.72^{\mathrm{a}}$ & $84.13 \pm 56.98$ & $58.52 \pm 29.76^{a}$ & $80.95 \pm 46.06$ & $61.18 \pm 41.74$ & 0.13 & 0.70 \\
\hline Vitamin C (mg) & $102.10 \pm 106.84$ & $112.68 \pm 177.30$ & $135.91 \pm 112.90$ & $54.59 \pm 47.03^{a}$ & $66.75 \pm 89.45$ & $173.42 \pm 236.61^{\mathrm{a}, \mathrm{c}}$ & 0.04 & 0.07 \\
\hline Vitamin E (mg) & $10.25 \pm 7.74$ & $6.91 \pm 6.72^{\mathrm{a}}$ & $10.98 \pm 7.83$ & $5.84 \pm 5.55^{\mathrm{a}}$ & $9.49 \pm 7.76$ & $8.03 \pm 7.73$ & 0.10 & 0.57 \\
\hline Folate (mcg) & $148.64 \pm 92.25$ & $108.66 \pm 68.03^{a}$ & $171.58 \pm 93.19$ & $84.57 \pm 42.83^{a}$ & $124.65 \pm 133.85$ & $133.85 \pm 80.47^{\mathrm{C}}$ & 0.12 & 0.67 \\
\hline
\end{tabular}

E-DII, Energy- adjusted Dietary Inflammatory Index; ${ }^{a}-P<0.05$;

statistic difference between baseline and after therapy of obese adolescents $(p<0.05)$;

${ }^{b}$ statistic difference between baseline of adolescents $\left(p<0.05^{c}\right)$ statistic difference between adolescents in the end of therapy;

${ }^{c}$ statistic difference between the groups in the end of therapy. 
TABLE 4 | Delta values of the parameters analyzed.

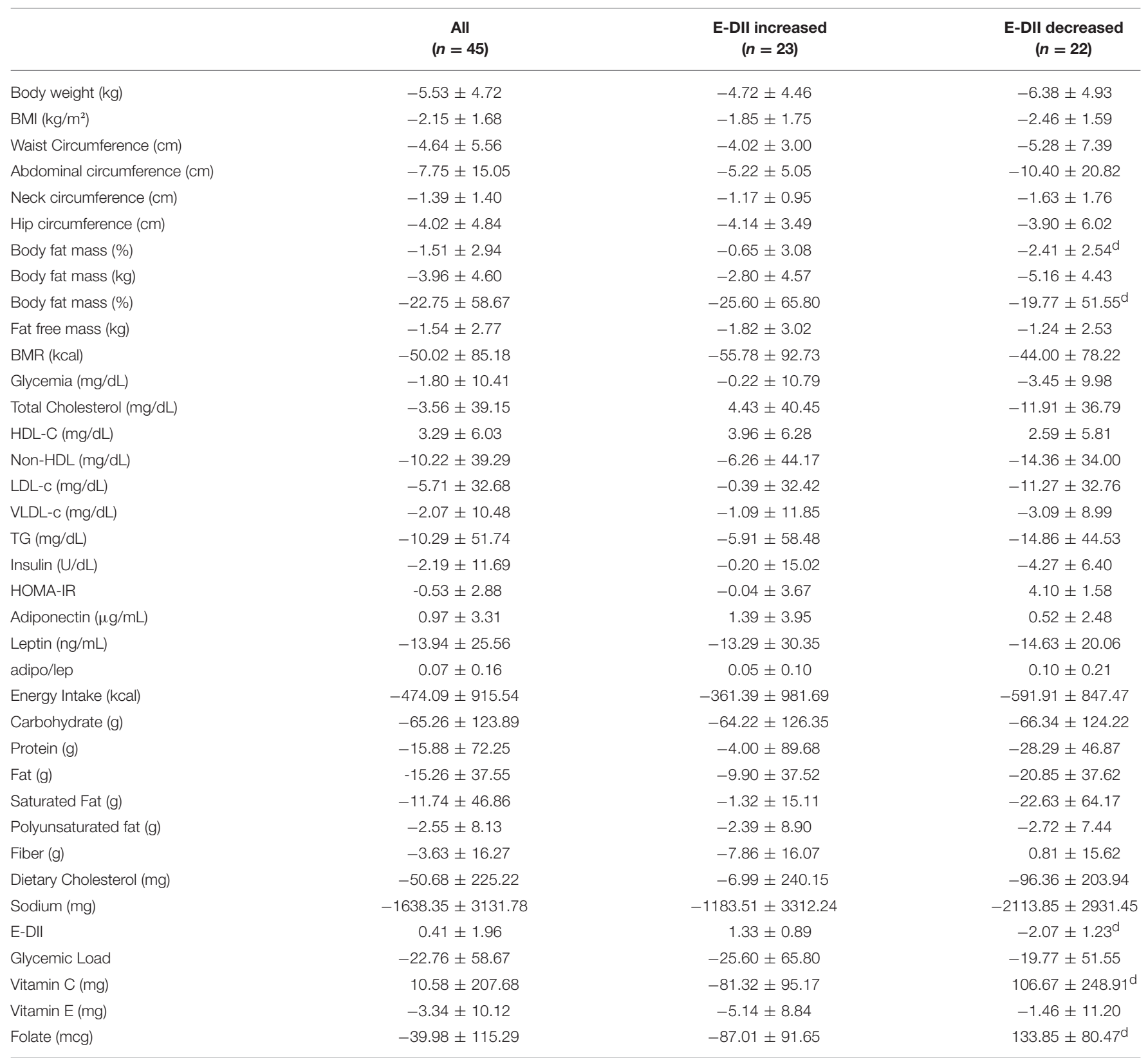

BMI (Body Mass Index), BMR (Basal Metabolic Rate); HDL-c, High-density lipoprotein cholesterol; LDL-c, Low-density lipoprotein cholesterol; VLDL-c, very low-density lipoprotein cholesterol; HOMA-IR, homeostasis model assessment insulin resistance; QUICKI, quantitative insulin-sensitivity check index; adipo/lep, adiponectin/leptin ratio;

${ }^{d}$ Difference statistic between delta $(\Delta)$ of DII increased and DII decreased.

For the components of dietary intake and E- DISCUSSION

DII, the deltas of Vitamin C, E, folate, and E-DII are statically different between adolescents that increased and decreased E-DII after the therapy (Table 4).

\section{Correlations Between Variables}

A negative correlation was found between $\Delta$ EDII and $\Delta$ Body fat mass $(\%)(-0.68 ; p=0.01)$ in the group of adolescents who increased the E-DII (Figure 2).
The present study indicates that long-term interdisciplinary therapy coupled with web-based health education can contribute to reducing obesity and associated inflammatory processes. Indeed, the E-DII can be used to ameliorate health conditions in adolescents with obesity. In fact, we consider E-DII relevant from a clinical point of view to improve biochemical parameters and inflammation parameters (leptin and adiponectin), as well as to help to attenuate cardiovascular risks. The present investigation indicates that adolescents who observed the dietary advice tended 
to have lower E-DII scores, and improvements in anthropometric and inflammatory parameters compared to their counterparts who continue to eat pro-inflammatory diet.

Previous studies showed that dietary pattern and obesity could induce inflammation and increase the risk for type 2 diabetes, cardiovascular disease, and metabolic syndrome (52-54). In the present investigation, we analyzed the possible relations between the E-DII score and cardiometabolic parameters in obese adolescents.

It was found an improvement in QUICKI after therapy occurred only in adolescents who decreased the E-DII, when the sample was analyzed according the E-DII response. QUICKI is a reliable index of insulin sensitivity (44). This same group decreased glycemia, VLDL-c, triglyceride, and HOMAIR. As previously demonstrated, insulin resistance is related to inflammatory processes, increasing risk of cardiovascular disease in obese adolescents $(36,55)$. It is important to know that these variables have been related to cardiovascular risks and metabolic syndrome, reinforcing the benefits of interdisciplinary therapy for the management of obesity and its related comorbidities in adolescents. Together, these results should be considered in the clinical practices.

Moreover, the therapy was effective at improving HDLc concentrations. High level of HDL-c may contribute to cardiometabolic protection, while low HDL-c correlates with metabolic syndrome in obese adolescent $(56,57)$.

Additionally, the present study showed negative correlation between $\Delta$ E-DII and $\Delta$ body fat mass (\%) in the group who increased the inflammatory diet profile, contributing to our understood to the importance of this E-DII in the clinical practice, as adjuvant in follow the patients to improve their health conditions.

Importantly our results show a reduction in total caloric intake as well as lower sodium, carbohydrate, and lipids intake by the end of the intervention of all adolescents. High calorie diet rich in lipids and micronutrients such as sodium can promote an increase in inflammatory indices of diet. It is well known that caloric intake higher than energy expenditure leads to body weight gain and fat deposition and that sodium intake is associated with metabolic disorders $(58,59)$.

Interesting, leptin is involved in the inflammatory process, and the state of hyperleptinemia may limit the adiponectin increase (9). Previous studies showed that adolescents undergoing long-term multidisciplinary therapy had significant reduction in leptin and increase in adiponectin concentration. These results are in line with our findings suggesting an attenuated inflammatory process after the proposed therapy. Although, the interdisciplinary intervention had been able to reduce hyperleptinemia state, children and adolescents should maintain healthy lifestyle to reach normal levels of this hormone $(35,60-63)$.

Hyperleptinemia was associated with hypoadiponectinemia, contributing to increased related inflammatory process obese adolescents $(11,35,61,64)$. Previous studies of this group showed that changes in body mass and BMI are positively correlated with decreased leptin concentration. It is important to note that a significant decrease in leptin concentration is observed when the adolescents reduced their body mass by at least 7\% (55). Confirming our hypothesis, we found a decrease in the leptin concentration and inversely an increase in adiponectin/leptin ratio in all analyzed adolescents after the therapy.

Moreover, we found an increase in adiponectin/leptin ratio and a decrease in leptin on the group of decreased E-DII. This ratio could be an atherosclerotic risk maker (65) while the adiponectin/leptin ratio could be a valuable complementary element in prediction and prevention of cardiovascular diseases and metabolic syndrome $(66,67)$, suggesting that our therapy was effective to improve these parameters.

Other interesting results from this study include the reduction of BMI and improved body composition. This corroborates a systematic review with obese adolescents that pointed out that a multicomponent therapy, including education health were effective in improving body composition and cardiometabolic biomarkers $(68,69)$.

Additionally, the interdisciplinary therapy was effective in reducing waist, neck, hip, and abdominal circumferences. These circumferences are positively correlated with cardiovascular risks, obesity, and metabolic syndrome; and the improvement of these anthropometric indicators are important in controlling obesity and cardiovascular diseases $(61,67,68,70-74)$.

Faced with the above results and the improvement of fat-free mass (\%), it is extremely important for health professionals to consider both the quantity and quality of the adolescent's diet as well as to encourage a change to healthy eating and active lifestyles, early in life, to prevent short-term and long-term morbidity and improve cardiometabolic parameters.

Some limitations of the present study are the lack of lean control group. Second, the Brazilian database is not complete with the 45 food parameters to calculate the EDII, moreover these analyses were exploratory. However, our study takes future directions to investigate the inflammatory diet and the impact on metabolic and inflammatory process of obesity.

In conclusion, the long-term interdisciplinary therapy combined with web-based health education were effective in improving inflammatory markers in adolescents with obesity. In addition, these results showed that in exploratory analyses, an improvement of inflammatory and cardiometabolic parameters occurred mainly when the E-DII improved an increased inflammatory profile diet, suggesting the relevance to stimulate anti-inflammatory diet habits as an effective strategy to prevent and treat obesity and related comorbidities in adolescents with obesity.

\section{ETHICS STATEMENT}

This study was carried out in accordance with the recommendations of Federal University to Sao Paulo ethics committee (\#0448/2018) with written informed consent from all subjects. All subjects gave written informed consent in accordance with the Declaration of Helsinki. The protocol was approved by the CEP UNIFESP. 


\section{AUTHOR CONTRIBUTIONS}

$\mathrm{YF}, \mathrm{AK}, \mathrm{SV}, \mathrm{AD}$, and LT designed the study. YF, AK, RC, $\mathrm{LO}, \mathrm{VB}$, and $\mathrm{AD}$ performed the experiments. YF, AK, RC, $\mathrm{DM}, \mathrm{LO}, \mathrm{VB}, \mathrm{AD}, \mathrm{DT}, \mathrm{NS}$, and $\mathrm{JH}$ analyzed the results and wrote the manuscript. LO, VB, NS, and $\mathrm{JH}$ contributed with reagents and analysis tools. All authors reviewed the manuscript.

\section{REFERENCES}

1. American Medical Association. American Medical Association House of Delegates. Policy Finder: Recognition of Obesity as a Disease (2013).

2. Hebebrand J, Holm J-C, Woodward E, Baker JL, Blaak E, Durrer Schutz $\mathrm{D}$, et al. A proposal of the european association for the study of obesity to improve the ICD-11 diagnostic criteria for obesity based on the three dimensions etiology, degree of adiposity and health risk. Obes Facts. (2017) 10:284-307. doi: 10.1159/000479208

3. Maffetone PB, Rivera-Dominguez I, Laursen PB. Overfat and underfat: new terms and definitions long overdue. Front Public Health. (2017) 2017:279. doi: 10.3389/fpubh.2016.00279

4. Instituto Brasileiro de Geografia e Estatística. Pesquisa de Orçamentos Familiares 2008-2009: Antropometria e Estado Nutricional de Crianças, Adolescentes e Adultos no Brasil. Rio de Janeiro: IBGE (2010).

5. World Health Organization (WHO). The World Health Report 2000. Obesity - preventing and managing the Global Epidemic II Series (2000).

6. World Health Organization (WHO). Global Health Risks: Mortality and Burden of Disease Attributable to Selected Major Risks (2009).

7. Pi-Sunyer X. The medical risks of obesity. Postgrad Med. (2009) 121:2133. doi: 10.3810/pgm.2009.11.2074

8. Mraz M, Haluzik M. The role of adipose tissue immune cells in obesity and low-grade inflammation. J Endocrinol. (2014) 222:11327. doi: 10.1530/JOE-14-0283

9. Ouchi N, Walsh K. Adiponectin as an anti-inflammatory factor. Clin Chim Acta. (2007) 380:24-30. doi: 10.1016/j.cca.2007.01.026

10. Auerbach P, Nordby P, Bendtsen LQ, Mehlsen JL, Basnet SK, Vestergaard $\mathrm{H}$, et al. Differential effects of endurance training and weight loss on plasma adiponectin multimers and adipose tissue macrophages in younger, moderately overweight men. Am J Physiol Regul Integr Comp Physiol. (2013) 305:490-8. doi: 10.1152/ajpregu.00575.2012

11. da Silva PL, de Mello MT, Cheik NC, Sanches PL, Piano A, Corgosinho FC, et al. The role of pro-inflammatory and anti-inflammatory adipokines on exercise-induced bronchospasm in obese adolescents undergoing treatment. Respir Care. (2012) 57:572-82. doi: 10.4187/respcare.01307

12. Polyzos SA, Aronis KN, Kountouras J, Raptis DD, Vasiloglou MF, Mantzoros CS. Circulating leptin in non-alcoholic fatty liver disease: a systematic review and meta-analysis. Diabetologia. (2016) 59:30-43. doi: 10.1007/s00125-015-3769-3

13. Schwartz MW, Seeley RJ, Zeltser LM, Drewnowski A, Ravussin E, Redman LM, et al. Obesity pathogenesis: an endocrine society scientific statement. Endocr Rev. (2017) 38:267-96. doi: 10.1210/er.2017-00111

14. Frühbeck G, Catalán V, Rodríguez A, Gómez-Ambrosi J. Adiponectinleptin ratio: a promising index to estimate adipose tissue dysfunction. Relation with obesity-associated cardiometabolic risk. Adipocyte. (2018) 7:5762. doi: 10.1080/21623945.2017.1402151

15. Geraldo JNM, Alfenas RDCG. Papel da dieta na prevenção e no controle da inflamação crônica: evidências atuais. Arq Bras Endocrinol Metabol. (2008) 52:951-67. doi: 10.1590/S0004-27302008000600006

16. Bakker GC, Van Erk MJ, Pellis L. An inflammatory dietary mix modulates inflammation and oxidative and metabolic stress in overweight men: a nutrigenomics approach. Am J Clin Nutr. (2010) 91:104459. doi: 10.3945/ajcn.2009.28822

17. Mahalle N, Kulkarni MV, Naik SS, Garg MK. Association of dietary factors with insulin resistance and inflammatory markers in subjects with diabetes

\section{FUNDING}

This work was supported by: Foundation of São Paulo Research-FAPESP [2017/07372-1; 2015/143099], National Council for Scientific and Technological Development-CNPq [300654/2013-8 and 409943/2016-9; 301322/2017-1], and Coordination of Higher Education Personnel Training-CAPES.

mellitus and coronary artery disease in Indian population. J Diabetes Complicat. (2014) 28:536-41. doi: 10.1016/j.jdiacomp.2012.09.008

18. Shivappa N, Schoenaker DA, Hebert JR, Mishra GD. Association between inflammatory potential of diet and risk of depression in middle-aged women: the Australian Longitudinal Study on Women's Health. Br J Nutr. (2016) 116:1077-86. doi: 10.1017/S000711451 6002853

19. Vázquez-Salas RA, Shivappa N, Galván-Portillo M, López-Carrillo L, Hébert JR, Torres-Sánchez L. Dietary inflammatory index and prostate cancer risk in a case-control study in Mexico. Br J Nutr. (2016) 116:194553. doi: 10.1017/S0007114516003986

20. Chassaing B, Vijay-Kumar M, Gewirtz AT. How diet can impact gut microbiota to promote or endanger health. Curr Opin Gastroenterol. (2017) 33:417-21. doi: 10.1097/MOG.0000000000000401

21. Erro R, Brigo F, Tamburin S, Zamboni M, Antonini A, Tinazzi M. Nutritional habits, risk, and progression of Parkinson disease. J Neurol. (2017) 2017:8639. doi: 10.1007/s00415-017-8639-0

22. Mazidi M, Shivappa N, Wirth MD, Hebert JR, Vatanparast H, Kengne AP. The association between dietary inflammatory properties and bone mineral density and risk of fracture in US adults. Eur J Clin Nutr. (2017) 2017:133. doi: 10.1038/ejcn.2017.133

23. Mehra K, Berkowitz A, Sanft T. Diet, physical activity, and body weight in cancer survivorship. Med Clin North Am. (2017) 101:115165. doi: 10.1016/j.mcna.2017.06.004

24. Shivappa N, Steck SE, Hurley TG, Hussey JR, Hébert JR. Designing and developing a literature-derived, populationbased dietary inflammatory index. Public Health Nutr. (2014) 17:1689-96. doi: 10.1017/S1368980013002115

25. Niclis C, Pou SA, Shivappa N, Hébert JR, Steck SE, Díaz MDP. Proinflammatory dietary intake is associated with increased risk of colorectal cancer: results of a case-control study in argentina using a multilevel modeling approach. Nutr Cancer. (2018) 70:61-8. doi: 10.1080/01635581.2018.13 97710

26. Andrade PA, Hermsdorff HHM, Leite JIA, Shivappa N, Hébert JR, Henriques $\mathrm{HKF}$, et al. Baseline pro-inflammatory diet is inversely associated with change in weight and body fat 6 months following-up to bariatric surgery. Obes Surg. (2018) 2018:3530. doi: 10.1007/s11695-018-3530-3

27. Carvalho C. The dietary inflammatory index and insulin resistance or metabolic syndrome in young adults. Nutrition. (2018) 2018:14. doi: 10.1016/j.nut.2018.07.014

28. Shivappa N, Hebert JR, Marcos A, Diaz LE, Gomez S, Nova E, et al. Association between dietary Inflammatory index and inflammatory markers in the HELENA study. Mol Nutr Food Res. (2017) 61:6. doi: 10.1002/mnfr.201600707

29. Shivappa N, Hebert JR, Rashidkhani B. Association between inflammatory potential of diet and stress levels in adolescent women in Iran. Arch Iran Med. (2017) 20:108-12.

30. Harris HR, Willett WC, Vaidya RL, Michels KB. An adolescent and early adulthood dietary pattern associated with inflammation and the incidence of breast cancer. Cancer Res. (2017) 77:117987. doi: 10.1158/0008-5472.CAN-16-2273

31. Sen S, Rifas-Shiman SL, Shivappa N, Wirth MD, Hebert JR, Gold DR, et al. Associations of prenatal and early life dietary inflammatory potential with childhood adiposity and cardiometabolic risk in Project Viva. Pediatr Obes. (2018) 13:292-300. doi: 10.1111/ijpo.12221 
32. Shivappa N, Hebert JR, Neshatbini Tehrani A, Bayzai B, Naja F, Rashidkhani B. A pro-inflammatory diet is associated with an increased odds of depression symptoms among iranian female adolescents: a cross-sectional study. Front Psychiatry. (2018) 29:400. doi: 10.3389/fpsyt.2018.00400

33. Rocha NP, Milagres LC, Longo GZ, Ribeiro AQ, Novaes JF. Association between dietary pattern and cardiometabolic risk in children and adolescents: a systematic review. J Pediatr. (2017) 93:214-22. doi: 10.1016/j.jped.2017.01.002

34. Silva DFO, Lyra CO, Lima SCVC. Dietary habits of adolescents and associated cardiovascular risk factors: a systematic review. Ciênc Saúde Coletiva. (2016) 21:1181-96. doi: 10.1590/1413-81232015214.08742015

35. Masquio DC, de Piano A, Campos RM, Sanches PL, Carnier J, Corgosinho FC, et al. The role of multicomponent therapy in the metabolic syndrome, inflammation and cardiovascular risk in obese adolescents. Br J Nutr. (2015) 113:1920-30. doi: 10.1017/S0007114515001129

36. de Sanches PL, Mello MT, Fonseca FA, Elias N, Piano AD, Carnier $\mathrm{J}$, et al. Insulin resistance can impair reduction on carotid intimamedia thickness in obese adolescents. Arq Bras Cardiol. (2012) 99:892-8. doi: 10.1590/S0066-782X2012005000077

37. Julian V, Thivel D, Pereira B, Costes F, Richard R, Duclos M. Improving peripheral and central vascular adjustments during exercise through a training program in adolescents with obesity. Obes Facts. (2016) 9:321-31. doi: 10.1159/000447456

38. Lopera CA, da Silva DF, Bianchini JA, Locateli JC, Moreira AC, Dada RP, et al. Effect of water- versus land-based exercise training as a component of a multidisciplinary intervention program for overweight and obese adolescents. Physiol Behav. (2016) 165:365-73. doi: 10.1016/j.physbeh.2016.08.019

39. Almeida SS, Corgosinho FC, Amorim CE, Gregnani MF, Campos RM, Masquio DC, et al. Different metabolic responses induced by long-term interdisciplinary therapy in obese adolescents related to ACE I/D polymorphism. J Renin Angiotensin Aldosterone Syst. (2017) 18:2. doi: 10.1177/1470320317703451

40. de Onis, M, Onyango AW, Borghi E, Siyam A, Nishida C, Siekmann J. Development of a WHO growth reference for school-aged children and adolescents. Bull World Health Organ. (2007) 85:660-7. doi: 10.2471/BLT.07.043497

41. Tanner JM, Whitehouse RH. Clinical longitudinal standards for height, weight, height velocity, weight velocity, and stages of puberty. Arch Dis Child. (1976) 51:170-9.

42. Schwimmer, JB, Deutsch R, Rauch JB, Behling C, Newbury R, Lavine JE. Obesity, insulin resistance, and other clinicopathological correlates of pediatric nonalcoholic fatty liver disease. J Pediatr. (2003) 143:5005. doi: 10.1067/S0022-3476(03)00325-1

43. American Diabetes Association. Diagnosis and classification of diabetes mellitus. Diabetes Care. (2015) 38:S8-16. doi: 10.2337/dc15-S005

44. Xavier HT, Izar MC, Faria Neto JR, Assad MH, Rocha VZ, Sposito AC, et al. V Diretriz Brasileira de Dislipidemias e Prevenção da Aterosclerose. Arquivos Brasil Cardiol. (2013) 101:1-20. doi: 10.5935/abc.2013S010

45. Matthews DR, Hosker JP, Rudenski AS, Naylor BA, Treacher DF, Turner RC. Homeostasis model assessment: insulin resistance and beta-cell function from fasting plasma glucose and insulin concentrations in man. Diabetologia. (1985) 28:412-9.

46. Katz A, Nambi SS, Mather K, Baron AD, Follmann DA, Sullivan G, et al. Quantitative insulin sensitivity check index: a simple, accurate method for assessing insulin sensitivity in humans. J Clin Endocrinol Metab. (2000) 85:2402-10. doi: 10.1210/jcem.85.7.6661

47. Dâmaso AR. Saber Emagrecer. São Paulo: Weight Science (2015).

48. Matsudo S, Araújo T, Matsudo V, Andrade D, Andrade E, Oliveira LC, et al. International physical activity questionnaire (IPAQ): study of validity and reliability in Brazil. Rev Brasil Atividade Física Saúde. (2001) 6:5-18.

49. Pinto AM, Gorin AA, Raynor HA, Tate DF, Fava JL, Wing RR. Successful weight-loss maintenance in relation to method of weight loss. Obesity. (2008) 16:2456-61. doi: 10.1038/oby.2008.364

50. Institute of Medicine (US) Subcommittee on Interpretation and Uses of Dietary Reference Intakes, Institute of Medicine (US) Standing Committee on the Scientific Evaluation of Dietary Reference Intakes. DRI Dietary Reference Intakes: Applications in Dietary Assessment. Washington, DC: National Academies Press (US) (2000).
51. Shivappa N, Hebert JR, Anderson LA, Shrubsole MJ, Murray LJ, Getty LB, et al. Dietary inflammatory index and risk of reflux oesophagitis, Barrett's oesophagus and oesophageal adenocarcinoma: a population-based case-control study. Br J Nutr. (2017) 117:132331. doi: 10.1017/S0007114517001131

52. Ozawa M, Shipley M, Kivimaki M, Singh-Manoux A, Brunner EJ. Dietary pattern, inflammation and cognitive decline: the Whitehall II prospective cohort study. Clin Nutr. (2017) 36:506-12. doi: 10.1016/j.clnu.2016.01.013

53. Casas R, Estruch R. Dietary patterns, foods, nutrients and chronic inflammatory disorders. Immunome Res. (2016) 12:122. doi: 10.4172/1745-7580.10000122

54. Calder, P, Ahluwalia, N, Brouns F. Dietary factors and low-grade inflammation in relation to overweight and obesity. Br J Nutr. (2011) 106:178. doi: 10.1017/S0007114511005460

55. Masquio DC, de Piano A, Sanches PL, Corgosinho FC, Campos RM, Carnier J, et al. The effect of weight loss magnitude on pro-/anti-inflammatory adipokines and carotid intima-media thickness in obese adolescents engaged in interdisciplinary weight loss therapy. Clin Endocrinol. (2013) 79:5564. doi: 10.1111/j.1365-2265.2012.04504.x

56. Welty FK. How do elevated triglycerides and low HDL-cholesterol affect inflammation and atherothrombosis? Curr Cardiol Rep. (2013) 15:400. doi: 10.1007/s11886-013-0400-4

57. Mahbuba S, Mohsin F, Rahat F, Nahar J, Begum T, Nahar N. Descriptive epidemiology of metabolic syndrome among obese adolescent population. Diabetes Metab Syndr. (2018) 2018:026. doi: 10.1016/j.dsx.2017.12.026

58. Reilly SM, Saltiel AR. Adapting to obesity with adipose tissue inflammation. Nat Ver Endocrinol. (2017) 13:633-43. doi: 10.1038/nrendo.2017.90

59. Oh SW, Koo HS, Han KH, Han SY, Chin HJ. Associations of sodium intake with obesity, metabolic disorder, and albuminuria according to age. PLoS ONE. (2017) 2017:e0188770. doi: 10.1371/journal.pone.0188770

60. Ackel-D’Elia C, Carnier J, Bueno CR Jr, Campos RM, Sanches PL, Clemente AP, et al. Effects of different physical exercises on leptin concentration in obese adolescents. Int J Sports Med. (2014) 35:164-71. doi: 10.1055/s-0033-1345128

61. de Carvalho-Ferreira JP, Masquio DC, da Silveira Campos RM, Dal Molin Netto B, Corgosinho FC, Sanches PL, et al. Is there a role for leptin in the reduction of depression symptoms during weight loss therapy in obese adolescent girls and boys? Peptides. (2015) 65:208. doi: 10.1016/j.peptides.2014.11.010

62. Gajewska J, Weker H, Ambroszkiewicz J, Chełchowska M, Wiech M, Laskowska-Klita $\mathrm{T}$. Changes in concentration of serum adiponectin multimeric forms following weight reduction programme in prepubertal obese children. Med Wieku Rozwoj. (2011) 15:298-305.

63. Blüher S, Panagiotou G, Petroff D, Markert J, Wagner A, Klemm T, et al. Effects of a 1-year exercise and lifestyle intervention on irisin, adipokines, and inflammatory markers in obese children. Obesity. (2014) 22:17018. doi: 10.1002/oby.20739

64. Huang F, Del-Río-Navarro BE, Pérez-Ontiveros JA, Ruiz-Bedolla E, SaucedoRamírez OJ, Villafaña $S$, et al. Effect of six-month lifestyle intervention on adiponectin, resistin and soluble tumor necrosis factor- $\alpha$ receptors in obese adolescents. Endocr J. (2014) 61:921-31. doi: 10.1507/endocrj. EJ14-0157

65. Selthofer-Relatić K, Radić R, Stupin A, Šišljagić V, Bošnjak I, Bulj N, et al Leptin/adiponectin ratio in overweight patients - gender differences. Diab Vasc Dis Res. (2018) 15:260-2. doi: 10.1177/1479164117752491

66. Lubkowska A, Radecka A, Bryczkowska I, Rotter I, Laszczyńska M, Dudzińska W. Serum adiponectin and leptin concentrations in relation to body fat distribution, hematological indices and lipid profile in humans. Int J Environ Res Public Health. (2015) 12:11528-48. doi: 10.3390/ijerph120911528

67. Vega GL, Grundy SM. Metabolic risk susceptibility in men is partially related to adiponectin/leptin ratio. J Obes. (2013) 2013:409679. doi: 10.1155/2013/409679

68. Rajjo T, Mohammed K, Alsawas M, Ahmed AT, Farah W, Asi N, et al. Treatment of pediatric obesity: an umbrella systematic review. J Clin Endocrinol Metab. (2017) 102:763-75. doi: 10.1210/jc.2016-2574

69. Mameli C, Krakauer JC, Krakauer NY, Bosetti A, Ferrari CM, Schneider L, et al. Effects of a multidisciplinary weight loss intervention in overweight and obese children and adolescents: 11 years of experience. PLoS ONE. (2017) 12:e0181095. doi: 10.1371/journal.pone.0181095 
70. Patnaik L, Pattnaik S, Rao EV, Sahu T. Validating neck circumference and waist circumference as anthropometric measures of overweight/obesity in adolescents. Indian Pediatr. (2017) 54:377-80.

71. Masquio DC, Ganen Ade P, Campos RM, Sanches Pde L, Corgosinho FC, Caranti D, et al. Cut-off values of waist circumference to predict metabolic syndrome in obese adolescents. Nutr Hosp. (2015) 31:154050. doi: 10.3305/nh.2015.31.4.8442

72. Mansour M, Nassef YE, Shady MA, Aziz AA, Malt HAE. Metabolic syndrome and cardiovascular risk factors in obese adolescent. Open Access Macedonian J Med Sci. (2016) 4:118-21. doi: 10.3889/oamjms.2016.037

73. Kurtoglu S, Hatipoglu N, Mazicioglu MM, Kondolot M. Neck circumference as a novel parameter to determine metabolic risk factors in obese children. Eur J Clin Invest. (2012) 42:623-30. doi: 10.1111/j.1365-2362.2011. 02627.x

74. Rajagopalan A, Balaji N. Association of neck circumference and obesity with blood pressure among adolescents in urban and rural population in North Tamil Nadu. J Nat Sci Biol Med. (2017) 8:144-9. doi: 10.4103/jnsbm.JNSBM_29_17
Conflict of Interest Statement: JH owns controlling interest in Connecting Health Innovations LLC (CHI), a company that has licensed the right to his invention of the dietary inflammatory index (DII) from the University of South Carolina in order to develop computer and smart phone applications for patient counseling and dietary intervention in clinical settings. NS is an employee of CHI.

The remaining authors declare that the research was conducted in the absence of any commercial or financial relationships that could be construed as a potential conflict of interest.

Copyright $\odot 2019$ Ferreira, Kravchychyn, Vicente, Campos, Tock, Oyama, Boldarine, Masquio, Thivel, Shivappa, Hébert and Dâmaso. This is an open-access article distributed under the terms of the Creative Commons Attribution License (CC BY). The use, distribution or reproduction in other forums is permitted, provided the original author(s) and the copyright owner(s) are credited and that the original publication in this journal is cited, in accordance with accepted academic practice. No use, distribution or reproduction is permitted which does not comply with these terms. 